\title{
Balance and Coordination Proficiency of Age-Matched Male and Female Children and Adolescents With Intellectual Disabilities
}

\author{
Ken Pitetti and Ruth Ann Miller \\ Wichita State University
}

\author{
E. Michael Loovis \\ Cleveland State University
}

\begin{abstract}
Male youth (8-18 years) with intellectual disability (ID) demonstrate motor proficiency below age-related competence capacities for typically developing youth. Whether below-criteria motor proficiency also exists for females with ID is not known. The purpose of this study was to determine if sex-specific differences exist in motor proficiency for youth with ID. The Bruininks-Oseretsky Test of Motor Proficiency was used to measure motor proficiency: six items for upper limb coordination, seven items for balance, and six items for bilateral coordination. One hundred and seventy-two (172) males and 85 females with ID but without Down syndrome were divided into five age groups for comparative purposes: 8-10,11-12,13-14, 15-16, and 17-21 years. Males scored sufficiently higher than females to suggest that sex data should not be combined to established Bruininks-Oseretsky Test of Motor Proficiency standards for upper limb coordination, balance, and bilateral coordination subtests.
\end{abstract}

Keywords: Bruininks-Oseretsky Test of Motor Proficiency, gross motor proficiency, sex-specific differences, variability of motor proficiency

Motor proficiency facilitates major body movements, such as walking, maintaining balance (BAL), coordination, and jumping. Adequate skill levels of motor proficiency/competence affect the general physical activity levels and physical fitness of children and adolescents (Hands, Larkin, Parker, Straker, \& Perry, 2009; Kambas et al., 2012; Rivilis et al., 2011; Wrotniak, Epstein, Dorn, Jones, \& Konditis, 2006) as well as health-related fitness (Robinson et al., 2015; Stodden, Gao, Goodway, \& Langendorfer, 2014). In addition, gross motor proficiency scores (i.e., BAL, upper body, and bilateral coordination [BLC]) have been used to identify relationships between motor proficiency and physical

Pitetti is with the Dept. of Physical Therapy, College of Health Professions, Wichita State University, Wichita, KS. Miller is with the Dept. of Electrical Engineering, College of Engineering, Wichita State University, Wichita, KS. Loovis is with the Dept. of Health and Human Performance, College of Education and Human Services, Cleveland State University, Cleveland, OH. Address author correspondence to Ken Pitetti at ken.pitetti@wichita.edu. 
activity (Wrotniak et al., 2006), generalized self-efficacy toward physical activity and participation in organized and free play sports (Cairney et al., 2005; Stodden et al., 2008), and overweight and obesity (Cairney, Hay, Faught, \& Hawes, 2005).

Pitetti, Miller, and Loovis (2017) recently assessed the gross motor proficiency of male children and adolescents with intellectual disability (ID) but without Down syndrome (DS) in areas of coordination and using the Bruininks-Oseretsky Test of Motor Proficiency-Second Edition (BOT-2; Bruininks \& Bruininks, 2005). The BOT-2 is utilized by researchers, occupational therapists, physical therapists, and developmental adapted physical education teachers as a standard for identifying motor deficits that require school-based therapy and assessment of therapeutic outcomes (Bruininks \& Bruininks, 2005). The study by Pitetti et al. (2017) reported that male youth with ID demonstrated below the age-related competence expected for typically developing (TD) children and adolescents without disabilities for static and dynamic BAL, BLC, and upper limb coordination (ULC). This finding has important implications in that identifying deficits in motor and coordination proficiency in youth with ID would improve corrective management to augment functional capacity in health-related physical activity (Krombholz, 2006; Wall, 2004; Watkinson et al., 2001; Wrotniak et al., 2006).

The BOT-2 (Bruininks \& Bruininks, 2005) also established sex-specific norms that represent normal differences in motor development between males and females. These sex-specific norms were meant to remove the possibility of interpreting low scores for a specific sex as a motor impairment. That is, females tend to score higher on subtest items of fine motor precision, fine motor integration, and manual dexterity; and males, especially beginning in the adolescent years, performed higher than females on subtest items in running speed and agility and strength (Bruininks \& Bruininks, 2005). However, the BOT-2 authors did not note sex-specific differences in their norm samples for BOT-2 subtests involving static and dynamic BAL, BLC, and ULC (Bruininks \& Bruininks, 2005). That is, male- and female-scaled scores for BAL, BLC, and ULC converted to similar total point scores.

The focus of the recent study by Pitetti et al. (2017) was to initiate the development of descriptive data to establish normative BOT-2 tables' specific to youth with ID. However, whether the BOT-2 normative data specific for male youth with ID can be applied to females with ID is not known. If motor impairment is identified relative to performance, and if performance in youth with ID is affected by differences in sex motor development, sex-specific norms should be established for this population. Therefore, the primary purpose of this study was to determine if age-matched sex-specific differences exist for static and dynamic BAL, bilateral, and upper body coordination in youth with ID, but without DS. A secondary purpose was to compare BAL and coordination motor capacities of study participants to criteria and characteristics established by the BOT-2 (Bruininks \& Bruininks, 2005) for youth with ID and TD youth.

\section{Methods}

\section{Participants}

A total of 172 males and 85 females (aged 8-21 years) with ID but without DS participated in data collection. Unique physical characteristics have been identified 
in youth with DS, which include excessive joint range of motion due to joint laxity (Pitetti, Miller, \& Beets, 2015); low muscle tone or hypotonia (Livingstone \& Birst, 1986); low peak aerobic capacity or exercise performance (Pitetti, Baynard, \& Agiovlasitis, 2013); and lower muscle strength (Cioni et al., 1994; Mercer \& Lewis, 2001). Therefore, youth with DS were not included in this study because these characteristics could affect motor proficiency.

Participants were engaged in general classroom activity before testing. Standing height (in centimeters) and weight (in kilograms) were obtained with participants not wearing shoes by using a portable stadiometer (Seca 214 portable height rod; Seca, Hamburg, Germany) and scale (Health o meter® scale, model no. HDM037-01; FitNow Inc., Boston, MA). Body mass index (weight [in kilograms] divided by height squared [in meters]) was calculated from measured data. Participant descriptive statistics at the time of testing are presented in Table 1.

The participants were from a Midwestern metropolitan area (population $\sim 350,000)$, and all participants had been classified as having mild ( $\mathrm{IQ}=50$ / 55-69) to moderate (IQ = 30/35-49) ID by school administrative personnel per the model of diagnosis by the American Association on Intellectual and Developmental Disabilities (2010).

Participants were chosen from a sample of convenience from local public schools and activity programs for youth with ID. The sample design was purposive in that participants had to meet the criterion for ID and could not have any known acute or chronic neuromuscular or joint conditions that would hinder their ability to perform evaluation maneuvers. Informed parental consent and participant verbal assent (i.e., answered "yes" when asked to participate) were obtained before

\section{Table 1 Descriptive Statistics, $M \pm S D$}

\begin{tabular}{|c|c|c|c|c|c|}
\hline & 8-10 Years & 11-12 Years & 13-14 Years & 15-16 Years & 17-21 Years \\
\hline \multicolumn{6}{|c|}{ Height (m) } \\
\hline M & $\begin{array}{c}(n=105) \\
1.42 \pm 0.16\end{array}$ & $\begin{array}{c}(n=135) \\
1.53 \pm 0.16\end{array}$ & $\begin{array}{c}(n=225) \\
1.62 \pm 0.13\end{array}$ & $\begin{array}{c}(n=219) \\
1.65 \pm 0.20\end{array}$ & $\begin{array}{c}(n=213) \\
1.69 \pm 0.11\end{array}$ \\
\hline $\mathrm{F}$ & $\begin{array}{c}(n=55) \\
1.35 \pm 0.16\end{array}$ & $\begin{array}{c}(n=103) \\
1.54 \pm 0.10\end{array}$ & $\begin{array}{c}(n=160) \\
1.59 \pm 0.17\end{array}$ & $\begin{array}{c}(n=133) \\
1.60 \pm 0.16\end{array}$ & $\begin{array}{c}(n=153) \\
1.60 \pm 0.09\end{array}$ \\
\hline \multicolumn{6}{|c|}{ Weight (kg) } \\
\hline M & $44.9 \pm 22.4$ & $58.2 \pm 26.3$ & $67.9 \pm 25.0$ & $73.7 \pm 26.1$ & $71.7 \pm 21.5$ \\
\hline $\mathrm{F}$ & $35.8 \pm 16.0$ & $58.4 \pm 22.6$ & $66.3 \pm 21.2$ & $66.3 \pm 13.1$ & $67.2 \pm 16.2$ \\
\hline \multicolumn{6}{|c|}{ Body mass index $\left(\mathrm{kg} / \mathrm{m}^{2}\right)$} \\
\hline M & $21.2 \pm 6.0$ & $23.5 \pm 6.8$ & $25.2 \pm 7.1$ & $26.2 \pm 7.7$ & $24.9 \pm 6.0$ \\
\hline $\mathrm{F}$ & $19.0 \pm 5.5$ & $24.3 \pm 7.8$ & $26.1 \pm 7.0$ & $25.8 \pm 4.4$ & $26.2 \pm 6.0$ \\
\hline \multicolumn{6}{|c|}{ Body mass percentiles $^{a}$} \\
\hline M & $>95$ & $>95$ & $>95$ & $>90$ & $>75$ \\
\hline $\mathrm{F}$ & $>85$ & $>95$ & $>95$ & $>90$ & $>85$ \\
\hline
\end{tabular}

Note. $\mathrm{M}=$ male; $\mathrm{F}=$ female.

${ }^{\mathrm{a}}$ National Center for Health Statistics in collaboration with the Centers for Disease Prevention and Health Promotion (2000). www.cdc.gov/growthcharts. 
participation in this study. Approval of this study was obtained from Wichita State University's institutional review board.

\section{Procedures and Instruments}

Items used for the BOT-2 subtest are presented in Table 2. Administration of each item followed the protocol established by Pitetti et al. $(2015,2017)$ for physical therapeutic evaluations of youth with ID using the BOT-2. That is, when testing for motor skills of youth with ID, it is important to "structure skill acquisition sessions to optimize the processing of task relevant information" (Elliott \& Bunn, 2004, p. 140). Accordingly, the target movement must be demonstrated in a manner that is understood clearly by a participant with ID. To address this concern, each item assessment incorporated a familiarization protocol. The familiarization protocol involved the following:

a. The movement was demonstrated to the participant twice;

b. The participant practiced the movement twice with prompting;

c. The participant practiced without prompting; and

d. The participant performed the movement twice without prompting with the best score used for data analysis.

The crucial element of the study was to ensure that the participant gave his or her best effort. That is, if during Steps 2 and 3, the evaluator determined that the participant either did not understand the instructions (i.e., could not perform the task) or did not want to perform the task, data from that specific participant were not included in the study. Tests were stopped if the participant reached the ceiling score (Table 2).

Two examiners were assigned per child with one demonstrating the item procedures and the other assuring safety (i.e., preventing falls) and prompting when necessary. Prompting consisted of manually correcting for the proper placement of limbs. Prompting did not occur when participants performed for data collection.

Examiners were predoctoral or doctoral students in physical therapy. The first author developed and supervised the preparative training of examiners for BOT-2 items and proctored all evaluations to insure proper measurements. Preparatory training protocol for examiners has been previously documented (Pitetti et al., 2015, 2017) and involved an instructional phase (i.e., peer-supportive learning) and a practice phase utilizing youth with ID recruited from local programs for persons with disabilities. High $(\geq 0.90)$ to good $(0.8-0.89)$ test-retest reliability (Lucas et al., 2013; Wuang, Lin, \& Su, 2009; Wuang \& Su, 2009) and excellent (intraclass correlation coefficient $>.75)$ to moderate $(.4 \geq$ intraclass correlation coefficient $\leq .75$ ) interrater reliability (Lucas et al., 2013; Pitetti et al., 2017) for the BOT-2 items used in this study have been established for children and adolescents with developmental disabilities.

\section{Data Analysis}

Data were collected over a 6-year period. Some participants were evaluated 2-6 times as they advanced in age group (Table 3). Previous work has established that BOT test scores assessed on a yearly basis are not influenced by a practice effect in youth with ID (Jobling, 1999; Wuang et al., 2009). 


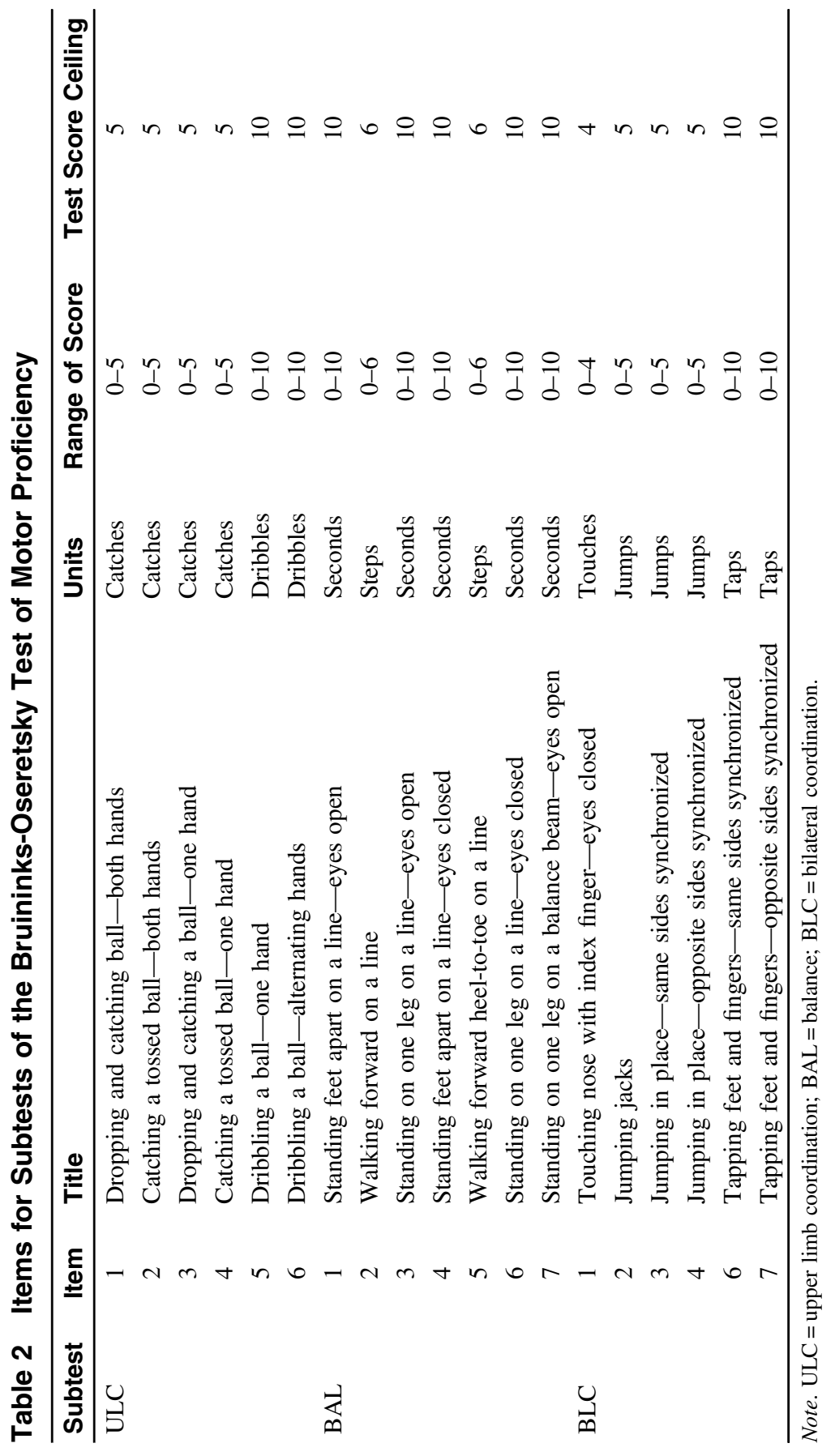


Table 3 Frequency of Yearly Assessment

\begin{tabular}{lc}
\hline Number of Times Evaluated & Participants \\
\hline One & 201 \\
Two & 117 \\
Three & 55 \\
Four & 36 \\
Five & 18 \\
Six & 10 \\
\hline
\end{tabular}

To organize and manage ongoing data collection, a Microsoft Access 2016 (Microsoft, Redmond, WA) database was developed. Queries were written, and means and $S D$ s were calculated for item scores in each subtest. These means and $S D$ s were calculated on data grouped per sex and age at the time of data collection.

An Access query was written to export a table containing the specific test (i.e., ULC-1, ULC-2, etc.) with raw test scores; participant ID; age of participant in years at the time of testing; the test score ceiling (Table 2); sex, and the percent of test score ceiling (test score/ceiling $\times 100$ ). To normalize subtest item scores for comparative purposes, all raw scores (i.e., steps, catches, touches, jumps, dribbles, BAL times) were converted to percent of test score ceiling (\%Ceiling). For instance, if a participant, when performing ULC-2 (catching a tossed ball-both hands), caught four of the five tossed balls, a raw score of " 4 " would be converted to a ceiling score of $80 \%(4 / 5 \times 100=80 \%$ ceiling $)$. The ceilings for all subtest items were established by the BOT-2 to represent the expected score for TD youth (i.e., no impairment exists; Bruininks \& Bruininks, 2005). Thus, scores were reported as percent of ceiling for three reasons: (a) it allowed for direct comparison of test results that had different raw ceiling scores; (b) it reduced the complexity of BOT-2 scoring whereby raw scores are combined to point scores and point scores are normalized to produce scale scores (Bruininks \& Bruininks, 2005); and (c) it permitted comparison of expected or norm scores of TD youth to the participants in this study.

A two-way analysis of variance (ANOVA) was performed using IBM SPSS software package (SPSS Inc., Chicago, IL) to determine if differences existed between sex, age groups (8-10, 11-12, 13-14, 15-16, and 17-21 years), and subtest items (Table 2: six items within subtest ULC, seven items within subtest BAL, and six items within subtest BLC) with \%Ceiling as the dependent variable. Assumptions for probability distributions independence were met except for normality and homogeneity. In that the tests were stopped once the participant reached ceiling scores, normal distribution could not be assumed due to ceiling effect (Vogt, 2005). Levene's test for equality of variance reported that the data did not meet homogeneity or equality of variances $(F=27.922 ; d f 1=206 ; d f 2=16,673 ; p<.000$; Levene, 1960). Although the data did not meet all assumptions for an ANOVA, the authors elected to perform a two-way ANOVA and set the level of significance to .001 to reduce the probability of a Type I error (i.e., to ensure that differences exist; Moore, McCabe, \& Craig, 2009). Values were reported as means and SDs.

If the two-way ANOVA identified statistically significant performance differences (\%Ceiling) within sex, age groups, and/or subtest, the following statistical 
tests were performed with level of significance held at .001 to reduce probability of a Type I error:

- Sex: A series of 95 separate $t$ tests was performed within each age group and subtest item (six items for ULC, seven items for BAL, and six items for BLC).

- Age groups: An ANOVA with post hoc Scheffe was performed to determine if motor performance differences (\% test score) existed for combined test items within each subtest test (six for ULC, seven for BAL, and six for BLC) across age groups $(8-10,11-12,13-14,15-16$, and 17-21 years) for males and females. A series of $t$ tests was performed for each age group within each subtest to determine if differences existed between sexes.

- Test items: An ANOVA with post hoc Scheffe from summary data of all age groups (combined 8-21 years) was performed to determine if motor performance (\%Ceiling scores) differences existed for test items within each subtest for males and females. A series of $t$ tests was performed for each test item within subtest to determine if differences existed between sexes.

- Finally, an ANOVA with post hoc Scheffe from summary data of combined age groups (8-21 years) and across subtest item scores (combining six items for BLC, seven items for BAL, and six items for ULC) was performed to determine if motor proficiency differed among subtests for males and females. A series of $t$ tests was performed for each test item within subtest to determine if differences existed between sexes.

\section{Results}

Tables 4-6 show results of subtest items by age and sex reported in percent ceiling (\%Ceiling) for ULC, BAL, and BLC, respectively. Means, SDs, and counts for raw scores by age and sex for BAL, ULC, and BLC subset items can be found in the Supplementary Tables 1-3 [available online], respectively. Over the six study period, of the total number of youth who were available from the sample of convenience, approximately $6 \%$ did not understand the instructions or did not want to perform the task.

Results of the SPSS two-way ANOVA analysis for interactions between subject effects and \%Ceiling as dependent variable are presented in Table 7 . The variables of sex, age group, and subtest items were significant in predicting participant performance $(p<.000)$. In addition, interaction factors of Sex $\times$ Subtest Items and Sex $\times$ Age Group were also significant in predicting participant performance $(p<.000)$. However, Subtest Items $\times$ Age Group $(p=.083)$ and Sex $\times$ Subtest Item $\times$ Age Group $(p=.117)$ did not meet the .001 significance level. That is, this data are best represented by a linear model in which there is no interaction between sex, age, and item tested (Sex $\times$ Age Group $\times$ Subtest) and also no interaction between subtest item and age group (Age Group $\times$ Subtest).

Therefore, given that significance was identified for the variables of sex, age group, and subtest items, the results are presented in the following manner: (a) performance differences between sexes in any of the 95 evaluations (19 Subtest Items $\times 5$ Age Groups); (b) performance differences between sexes 
Table 4 ULC Percent Ceiling

\begin{tabular}{|c|c|c|c|c|c|}
\hline & 8-10 Years & 11-12 Years & 13-14 Years & 15-16 Years & 17-21 Years \\
\hline \multicolumn{6}{|c|}{ ULC-1 } \\
\hline M & $\begin{array}{c}(n=69) \\
92.5 \pm 17.2\end{array}$ & $\begin{array}{c}(n=93) \\
91.8 \pm 17.8\end{array}$ & $\begin{array}{c}(n=149) \\
93.8 \pm 17.8\end{array}$ & $\begin{array}{c}(n=138) \\
94.2 \pm 17.1\end{array}$ & $\begin{array}{c}(n=138) \\
91.6 \pm 21.6\end{array}$ \\
\hline $\mathrm{F}$ & $\begin{array}{c}(n=35) \\
80.0 \pm 29.5\end{array}$ & $\begin{array}{c}(n=65) \\
91.7 \pm 18.0\end{array}$ & $\begin{array}{c}(n=95) \\
89.6 \pm 24.0\end{array}$ & $\begin{array}{c}(n=83) \\
92.3 \pm 21.1\end{array}$ & $\begin{array}{c}(n=96) \\
89.0 \pm 24.1\end{array}$ \\
\hline \multicolumn{6}{|c|}{ ULC-2 } \\
\hline M & $\begin{array}{c}(n=67) \\
80.9 \pm 28.8^{*}\end{array}$ & $\begin{array}{c}(n=92) \\
82.0 \pm 30.9\end{array}$ & $\begin{array}{c}(n=146) \\
85.6 \pm 26.0\end{array}$ & $\begin{array}{c}(n=140) \\
87.9 \pm 25.7\end{array}$ & $\begin{array}{c}(n=140) \\
87.7 \pm 27.4\end{array}$ \\
\hline $\mathrm{F}$ & $\begin{array}{c}(n=31) \\
56.8 \pm 40.0\end{array}$ & $\begin{array}{c}(n=62) \\
80.6 \pm 29.9\end{array}$ & $\begin{array}{c}(n=99) \\
89.3 \pm 21.1\end{array}$ & $\begin{array}{c}(n=94) \\
93.6 \pm 17.1\end{array}$ & $\begin{array}{c}(n=107) \\
88.2 \pm 22.1\end{array}$ \\
\hline \multicolumn{6}{|c|}{ ULC-3 } \\
\hline M & $\begin{array}{c}(n=60) \\
89.3 \pm 24.8^{*}\end{array}$ & $\begin{array}{c}(n=81) \\
93.8 \pm 18.9\end{array}$ & $\begin{array}{c}(n=128) \\
93.4 \pm 20.3\end{array}$ & $\begin{array}{c}(n=119) \\
96.0 \pm 15.1\end{array}$ & $\begin{array}{c}(n=122) \\
93.4 \pm 20.3\end{array}$ \\
\hline $\mathrm{F}$ & $\begin{array}{c}(n=30) \\
55.3 \pm 44.0\end{array}$ & $\begin{array}{c}(n=56) \\
84.6 \pm 28.0\end{array}$ & $\begin{array}{c}(n=89) \\
86.5 \pm 26.4\end{array}$ & $\begin{array}{c}(n=86) \\
90.0 \pm 24.1\end{array}$ & $\begin{array}{c}(n=97) \\
87.2 \pm 26.3\end{array}$ \\
\hline \multicolumn{6}{|c|}{ ULC-4 } \\
\hline M & $\begin{array}{c}(n=41) \\
55.6 \pm 36.5\end{array}$ & $\begin{array}{c}(n=58) \\
57.6 \pm 35.8\end{array}$ & $\begin{array}{c}(n=94) \\
60.4 \pm 39.3\end{array}$ & $\begin{array}{c}(n=82) \\
62.4 \pm 37.4\end{array}$ & $\begin{array}{c}(n=88) \\
58.9 \pm 48.6\end{array}$ \\
\hline $\mathrm{F}$ & $\begin{array}{c}(n=20) \\
35.0 \pm 38.7\end{array}$ & $\begin{array}{c}(n=37) \\
56.2 \pm 40.0\end{array}$ & $\begin{array}{c}(n=56) \\
56.4 \pm 39.4\end{array}$ & $\begin{array}{c}(n=59) \\
68.5 \pm 36.0\end{array}$ & $\begin{array}{c}(n=67) \\
60.3 \pm 38.1\end{array}$ \\
\hline \multicolumn{6}{|c|}{ ULC-5 } \\
\hline M & $\begin{array}{c}(n=70) \\
75.7 \pm 30.5^{*}\end{array}$ & $\begin{array}{c}(n=98) \\
81.9 \pm 26.7\end{array}$ & $\begin{array}{c}(n=152) \\
82.2 \pm 25.6\end{array}$ & $\begin{array}{c}(n=140) \\
83.5 \pm 24.9\end{array}$ & $\begin{array}{c}(n=138) \\
79.4 \pm 28.5\end{array}$ \\
\hline $\mathrm{F}$ & $\begin{array}{c}(n=36) \\
44.7 \pm 45.1\end{array}$ & $\begin{array}{c}(n=66) \\
70.6 \pm 35.9\end{array}$ & $\begin{array}{c}(n=95) \\
75.7 \pm 30.4\end{array}$ & $\begin{array}{c}(n=86) \\
78.6 \pm 28.1\end{array}$ & $\begin{array}{c}(n=94) \\
75.9 \pm 32.3\end{array}$ \\
\hline \multicolumn{6}{|c|}{ ULC-6 } \\
\hline M & $\begin{array}{c}(n=67) \\
66.6 \pm 36.3 *\end{array}$ & $\begin{array}{c}(n=89) \\
71.0 \pm 33.0\end{array}$ & $\begin{array}{c}(n=145) \\
68.8 \pm 32.8\end{array}$ & $\begin{array}{c}(n=138) \\
70.4 \pm 32.8\end{array}$ & $\begin{array}{c}(n=136) \\
71.9 \pm 32.2\end{array}$ \\
\hline $\mathrm{F}$ & $\begin{array}{c}(n=32) \\
31.3 \pm 34.3\end{array}$ & $\begin{array}{c}(n=58) \\
60.0 \pm 36.2\end{array}$ & $\begin{array}{c}(n=95) \\
61.8 \pm 35.4\end{array}$ & $\begin{array}{c}(n=90) \\
68.4 \pm 33.2\end{array}$ & $\begin{array}{c}(n=104) \\
63.1 \pm 350.8\end{array}$ \\
\hline
\end{tabular}

Note. $\mathrm{ULC}=$ upper limb coordination; $\mathrm{M}=$ male; $\mathrm{F}=$ female.

*Males significantly $(p>.001)$ higher than females.

for the combined subtest scores (ULC, BAL, and BLC) for each of the five age groups; and (c) performance differences between sexes whom combining all age groups for each subtest item.

\section{Sex $\times$ Subtest Items $\times$ Age Groups}

When considering the 95 comparisons (19 Subtest Items $\times 5$ Age Groups), only four subtest items for one age group (8-10 years) demonstrated a sex difference with males performing significantly better than females (see Table 4): 


\section{Table 5 BAL Percent Ceiling}

\begin{tabular}{|c|c|c|c|c|c|}
\hline & 8-10 Years & 11-12 Years & 13-14 Years & 15-16 Years & 17-21 Years \\
\hline \multicolumn{6}{|c|}{ BAL-1 } \\
\hline M & $\begin{array}{c}(n=58) \\
88.4 \pm 25.2\end{array}$ & $\begin{array}{c}(n=79) \\
89.4 \pm 24.1\end{array}$ & $\begin{array}{c}(n=125) \\
91.3 \pm 23.7\end{array}$ & $\begin{array}{c}(n=116) \\
95.1 \pm 18.5\end{array}$ & $\begin{array}{c}(n=120) \\
95.0 \pm 19.5\end{array}$ \\
\hline $\mathrm{F}$ & $\begin{array}{c}(n=29) \\
83.7 \pm 27.0\end{array}$ & $\begin{array}{c}(n=57) \\
87.2 \pm 25.0\end{array}$ & $\begin{array}{c}(n=85) \\
92.5 \pm 21.9\end{array}$ & $\begin{array}{c}(n=86) \\
94.1 \pm 20.2\end{array}$ & $\begin{array}{c}(n=96) \\
92.3 \pm 22.7\end{array}$ \\
\hline \multicolumn{6}{|c|}{ BAL-2 } \\
\hline M & $\begin{array}{c}(n=57) \\
91.8 \pm 20.2\end{array}$ & $\begin{array}{c}(n=81) \\
89.3 \pm 25.4\end{array}$ & $\begin{array}{c}(n=128) \\
90.5 \pm 23.9\end{array}$ & $\begin{array}{c}(n=117) \\
93.1 \pm 21.9\end{array}$ & $\begin{array}{c}(n=118) \\
93.9 \pm 21.6\end{array}$ \\
\hline $\mathrm{F}$ & $\begin{array}{c}(n=27) \\
77.8 \pm 41.1\end{array}$ & $\begin{array}{c}(n=56) \\
93.4 \pm 26.0\end{array}$ & $\begin{array}{c}(n=89) \\
91.9 \pm 26.1\end{array}$ & $\begin{array}{c}(n=86) \\
96.5 \pm 14.7\end{array}$ & $\begin{array}{c}(n=94) \\
90.6 \pm 22.4\end{array}$ \\
\hline \multicolumn{6}{|c|}{ BAL-3 } \\
\hline M & $\begin{array}{c}(n=57) \\
77.6 \pm 33.0\end{array}$ & $\begin{array}{c}(n=79) \\
72.6 \pm 32.3\end{array}$ & $\begin{array}{c}(n=125) \\
79.7 \pm 29.5\end{array}$ & $\begin{array}{c}(n=116) \\
85.8 \pm 25.5\end{array}$ & $\begin{array}{c}(n=124) \\
82.6 \pm 30.0\end{array}$ \\
\hline $\mathrm{F}$ & $\begin{array}{c}(n=28) \\
65.8 \pm 35.2\end{array}$ & $\begin{array}{c}(n=54) \\
73.3 \pm 31.7\end{array}$ & $\begin{array}{c}(n=85) \\
79.3 \pm 29.2\end{array}$ & $\begin{array}{c}(n=86) \\
83.6 \pm 26.1\end{array}$ & $\begin{array}{c}(n=95) \\
73.6 \pm 33.1\end{array}$ \\
\hline \multicolumn{6}{|c|}{ BAL-4 } \\
\hline M & $\begin{array}{c}(n=57) \\
69.8 \pm 34.8\end{array}$ & $\begin{array}{c}(n=76) \\
69.2 \pm 34.0\end{array}$ & $\begin{array}{c}(n=135) \\
69.0 \pm 31.4\end{array}$ & $\begin{array}{c}(n=123) \\
74.1 \pm 28.9\end{array}$ & $\begin{array}{c}(n=122) \\
77.9 \pm 30.2\end{array}$ \\
\hline $\mathrm{F}$ & $\begin{array}{c}(n=31) \\
47.0 \pm 32.8\end{array}$ & $\begin{array}{c}(n=56) \\
69.5 \pm 34.1\end{array}$ & $\begin{array}{c}(n=86) \\
67.3 \pm 34.9\end{array}$ & $\begin{array}{c}(n=78) \\
74.4 \pm 31.2\end{array}$ & $\begin{array}{c}(n=92) \\
67.2 \pm 35.3\end{array}$ \\
\hline \multicolumn{6}{|c|}{ BAL-5 } \\
\hline M & $\begin{array}{c}(n=68) \\
68.9 \pm 36.9\end{array}$ & $\begin{array}{c}(n=86) \\
67.1 \pm 37.9\end{array}$ & $\begin{array}{c}(n=150) \\
74.1 \pm 35.1\end{array}$ & $\begin{array}{c}(n=137) \\
79.4 \pm 31.7\end{array}$ & $\begin{array}{c}(n=133) \\
76.8 \pm 37.0\end{array}$ \\
\hline $\mathrm{F}$ & $\begin{array}{c}(n=26) \\
62.8 \pm 53.4\end{array}$ & $\begin{array}{c}(n=57) \\
72.8 \pm 39.7\end{array}$ & $\begin{array}{c}(n=88) \\
64.2 \pm 44.9\end{array}$ & $\begin{array}{c}(n=76) \\
74.3 \pm 37.0\end{array}$ & $\begin{array}{c}(n=96) \\
62.3 \pm 41.6\end{array}$ \\
\hline \multicolumn{6}{|c|}{ BAL-6 } \\
\hline M & $\begin{array}{c}(n=37) \\
56.9 \pm 33.4\end{array}$ & $\begin{array}{c}(n=55) \\
51.3 \pm 30.4\end{array}$ & $\begin{array}{c}(n=93) \\
47.1 \pm 28.4\end{array}$ & $\begin{array}{c}(n=77) \\
56.9 \pm 30.1\end{array}$ & $\begin{array}{c}(n=83) \\
54.2 \pm 31.3\end{array}$ \\
\hline $\mathrm{F}$ & $\begin{array}{c}(n=18) \\
39.6 \pm 31.4\end{array}$ & $\begin{array}{c}(n=37) \\
47.7 \pm 33.7\end{array}$ & $\begin{array}{c}(n=56) \\
49.5 \pm 32.0\end{array}$ & $\begin{array}{c}(n=58) \\
54.5 \pm 31.2\end{array}$ & $\begin{array}{c}(n=63) \\
47.9 \pm 31.8\end{array}$ \\
\hline \multicolumn{6}{|c|}{ BAL-7 } \\
\hline M & $\begin{array}{c}(n=62) \\
60.9 \pm 33.0\end{array}$ & $\begin{array}{c}(n=84) \\
52.8 \pm 33.1\end{array}$ & $\begin{array}{c}(n=146) \\
51.1 \pm 33.0\end{array}$ & $\begin{array}{c}(n=136) \\
52.1 \pm 32.0\end{array}$ & $\begin{array}{c}(n=137) \\
52.2 \pm 31.3\end{array}$ \\
\hline $\mathrm{F}$ & $\begin{array}{c}(n=31) \\
47.6 \pm 32.6\end{array}$ & $\begin{array}{c}(n=61) \\
47.2 \pm 31.8\end{array}$ & $\begin{array}{c}(n=93) \\
42.2 \pm 31.2\end{array}$ & $\begin{array}{c}(n=81) \\
48.3 \pm 33.6\end{array}$ & $\begin{array}{c}(n=94) \\
38.5 \pm 31.7\end{array}$ \\
\hline
\end{tabular}

Note. $\mathrm{BAL}=$ balance; $\mathrm{M}=$ male $; \mathrm{F}=$ female.

- ULC-2 (males $=80.9 \pm 28.8$; females $=56.8 \pm 40.0$ ),

- ULC-3 (males = 89.3 \pm 24.3 ; females $55.3 \pm 44.0$ ),

- ULC-5 (males $=75.7 \pm 30.5$; females $=44.7 \pm 45.1$ ), and

- ULC-6 $($ males $=66.6 \pm 36.3$; females $=31.3 \pm 34.3$ ). 


\section{Table 6 BLC Percent Ceiling}

\begin{tabular}{|c|c|c|c|c|c|}
\hline & 8-10 Years & 11-12 Years & 13-14 Years & 15-16 Years & 17-21 Years \\
\hline \multicolumn{6}{|c|}{ BLC-1 } \\
\hline M & $\begin{array}{c}(n=71) \\
90.1 \pm 24.8\end{array}$ & $\begin{array}{c}(n=87) \\
90.2 \pm 24.8\end{array}$ & $\begin{array}{c}(n=155) \\
94.4 \pm 18.6\end{array}$ & $\begin{array}{c}(n=147) \\
93.7 \pm 19.8\end{array}$ & $\begin{array}{c}(n=143) \\
96.0 \pm 17.2\end{array}$ \\
\hline $\mathrm{F}$ & $\begin{array}{c}(n=37) \\
75.7 \pm 29.7\end{array}$ & $\begin{array}{c}(n=60) \\
94.6 \pm 20.6\end{array}$ & $\begin{array}{c}(n=89) \\
92.7 \pm 27.5\end{array}$ & $\begin{array}{c}(n=78) \\
96.8 \pm 21.1\end{array}$ & $\begin{array}{c}(n=99) \\
93.7 \pm 20.0\end{array}$ \\
\hline \multicolumn{6}{|c|}{ BLC-2 } \\
\hline M & $\begin{array}{c}(n=59) \\
76.6 \pm 36.0\end{array}$ & $\begin{array}{c}(n=81) \\
75.6 \pm 36.2\end{array}$ & $\begin{array}{c}(n=128) \\
88.6 \pm 26.5\end{array}$ & $\begin{array}{c}(n=118) \\
89.2 \pm 27.8\end{array}$ & $\begin{array}{c}(n=122) \\
81.3 \pm 35.4\end{array}$ \\
\hline $\mathrm{F}$ & $\begin{array}{c}(n=27) \\
65.9 \pm 40.3\end{array}$ & $\begin{array}{c}(n=61) \\
83.5 \pm 33.8\end{array}$ & $\begin{array}{c}(n=85) \\
81.4 \pm 36.0\end{array}$ & $\begin{array}{c}(n=82) \\
82.9 \pm 33.6\end{array}$ & $\begin{array}{c}(n=91) \\
77.4 \pm 43.1\end{array}$ \\
\hline \multicolumn{6}{|c|}{ BLC-3 } \\
\hline M & $\begin{array}{c}(n=58) \\
71.0 \pm 40.7\end{array}$ & $\begin{array}{c}(n=78) \\
76.4 \pm 36.1\end{array}$ & $\begin{array}{c}(n=128) \\
88.4 \pm 37.4\end{array}$ & $\begin{array}{c}(n=116) \\
91.0 \pm 22.9\end{array}$ & $\begin{array}{c}(n=119) \\
88.4 \pm 25.8\end{array}$ \\
\hline $\mathrm{F}$ & $\begin{array}{c}(n=23) \\
57.4 \pm 46.4\end{array}$ & $\begin{array}{c}(n=49) \\
78.0 \pm 39.8\end{array}$ & $\begin{array}{c}(n=83) \\
73.0 \pm 41.8\end{array}$ & $\begin{array}{c}(n=79) \\
78.7 \pm 37.4\end{array}$ & $\begin{array}{c}(n=91) \\
70.1 \pm 47.6\end{array}$ \\
\hline \multicolumn{6}{|c|}{ BLC-4 } \\
\hline M & $\begin{array}{c}(n=65) \\
47.4 \pm 43.2\end{array}$ & $\begin{array}{c}(n=84) \\
52.4 \pm 43.5\end{array}$ & $\begin{array}{c}(n=149) \\
60.9 \pm 43.1\end{array}$ & $\begin{array}{c}(n=140) \\
65.7 \pm 41.4\end{array}$ & $\begin{array}{c}(n=138) \\
62.8 \pm 45.4\end{array}$ \\
\hline $\mathrm{F}$ & $\begin{array}{c}(n=30) \\
44.7 \pm 46.3\end{array}$ & $\begin{array}{c}(n=56) \\
51.1 \pm 44.3\end{array}$ & $\begin{array}{c}(n=84) \\
46.9 \pm 44.4\end{array}$ & $\begin{array}{c}(n=72) \\
53.3 \pm 43.0\end{array}$ & $\begin{array}{c}(n=88) \\
46.4 \pm 45.4\end{array}$ \\
\hline \multicolumn{6}{|c|}{ BLC-6 } \\
\hline M & $\begin{array}{c}(n=64) \\
87.2 \pm 26.6\end{array}$ & $\begin{array}{c}(n=86) \\
93.7 \pm 15.9\end{array}$ & $\begin{array}{c}(n=154) \\
96.9 \pm 14.3\end{array}$ & $\begin{array}{c}(n=148) \\
95.8 \pm 17.4\end{array}$ & $\begin{array}{c}(n=141) \\
90.3 \pm 23.7\end{array}$ \\
\hline $\mathrm{F}$ & $\begin{array}{c}(n=29) \\
75.5 \pm 29.9\end{array}$ & $\begin{array}{c}(n=59) \\
92.4 \pm 22.5\end{array}$ & $\begin{array}{c}(n=89) \\
88.3 \pm 24.1\end{array}$ & $\begin{array}{c}(n=80) \\
90.1 \pm 31.6\end{array}$ & $\begin{array}{c}(n=99) \\
89.9 \pm 21.9\end{array}$ \\
\hline \multicolumn{6}{|c|}{ BLC-7 } \\
\hline M & $\begin{array}{c}(n=54) \\
63.3 \pm 43.0\end{array}$ & $\begin{array}{c}(n=77) \\
72.1 \pm 88.2\end{array}$ & $\begin{array}{c}(n=126) \\
81.8 \pm 31.6\end{array}$ & $\begin{array}{c}(n=119) \\
84.3 \pm 30.3\end{array}$ & $\begin{array}{c}(n=120) \\
80.6 \pm 33.5\end{array}$ \\
\hline $\mathrm{F}$ & $\begin{array}{c}(n=22) \\
66.8 \pm 41.0\end{array}$ & $\begin{array}{c}(n=52) \\
72.1 \pm 40.7\end{array}$ & $\begin{array}{c}(n=81) \\
73.1 \pm 40.4\end{array}$ & $\begin{array}{c}(n=84) \\
75.1 \pm 39.8\end{array}$ & $\begin{array}{c}(n=91) \\
72.2 \pm 43.4\end{array}$ \\
\hline
\end{tabular}

Note $. \mathrm{BLC}=$ bilateral coordination; $\mathrm{M}=$ male $; \mathrm{F}=$ female.

Significant differences were not seen between sex in all other combination of subtest items and age group.

\section{Sex $\times$ Combined Subtest Scores $\times$ Age Groups}

- ULC: Subtest means for combined items are found in Figure 1.

Males: No significant differences were seen across age groups.

Females: The 8- to 10-year age group was significantly lower than the other four age groups. 
Table 7 Results of Two-Way ANOVA for Interactions Between Gender, Age Groups, and Subtest Items

\begin{tabular}{lccc}
\hline Source & $\boldsymbol{d f}$ & $\boldsymbol{F}$ & $\boldsymbol{p}$ \\
\hline Sex & 1 & 131.741 & .000 \\
Subtest item & 21 & 172.071 & .000 \\
Age Group & 4 & 40.01 & .000 \\
Sex $\times$ Subtest Item & 38 & 2.587 & .000 \\
Sex $\times$ Age Group & 4 & 12.745 & .000 \\
Subtest Item $\times$ Age Group & 80 & 1.227 & .083 \\
Sex $\times$ Subtest Item $\times$ Age Group & 76 & 1.197 & .117 \\
Error & 16,673 & & \\
\hline
\end{tabular}

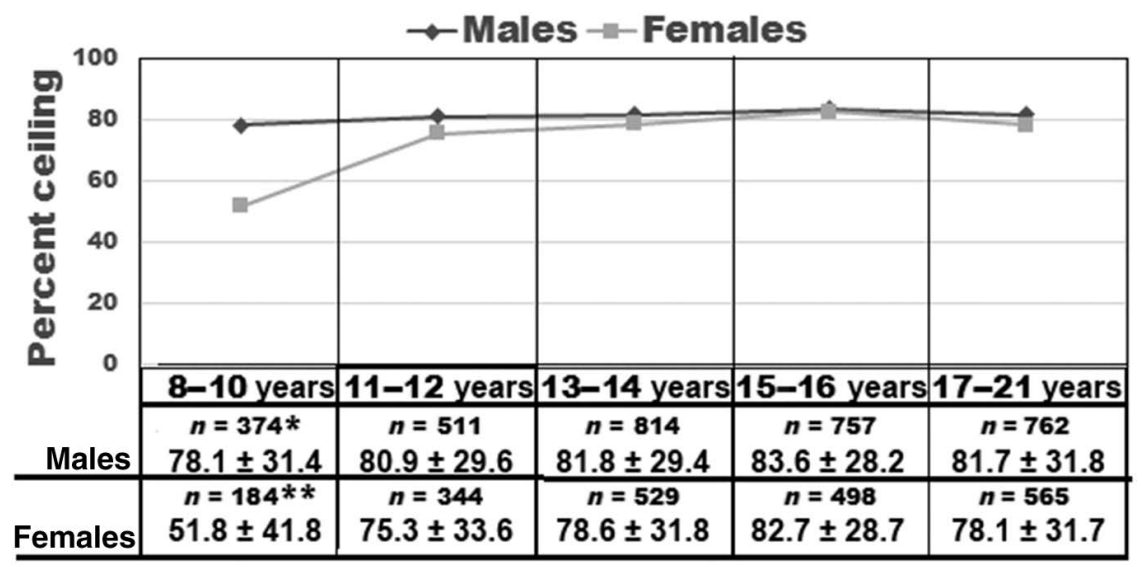

Figure 1 - ULC subtest for combined items (6) for each age group. *Males significantly $(p<.001)$ higher than females in 8 - to 10 -year age group. **Females in the 8 - to 10 -year age group significantly $(p<.001)$ lower than all other female age groups. ULC $=$ upper limb coordination.

Sexes: Male in 8- to 10-year age group was higher than female in 8- to 10-year age group. No significant difference between sexes was seen in the other age groups.

- BAL: Subtest means for combined items are found in Figure 2.

Males: No significant differences were seen across age groups.

Females: The 8- to 10-year age group was significantly lower than 15 - to 16-year age group.

Sexes: Male in 8-to 10-year and 17- to 21-year age groups was higher than their respective female age groups.

- BLC: Subtest means for combined items are found in Figure 3. 


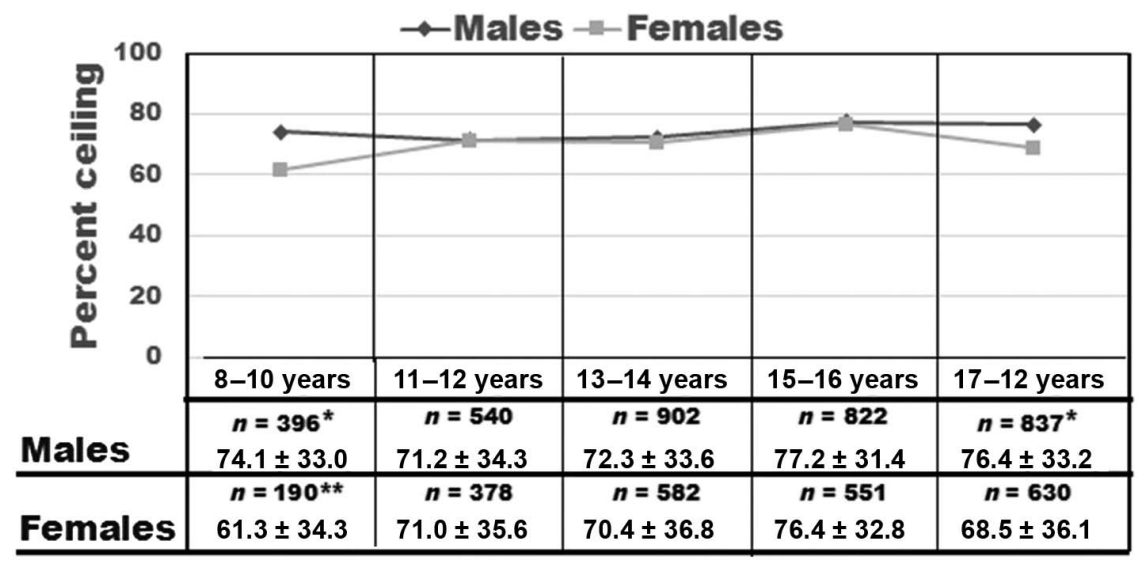

Figure 2 - BAL subtest for combined items (7) for each age group. *Males significantly $(p<.001)$ higher than females in 8- to 10-year age group and 17- to 21-year age group. **Females in the 8 - to 10 -year age group significantly $(p<.001)$ lower than all other female age groups. $\mathrm{BAL}=$ balance.

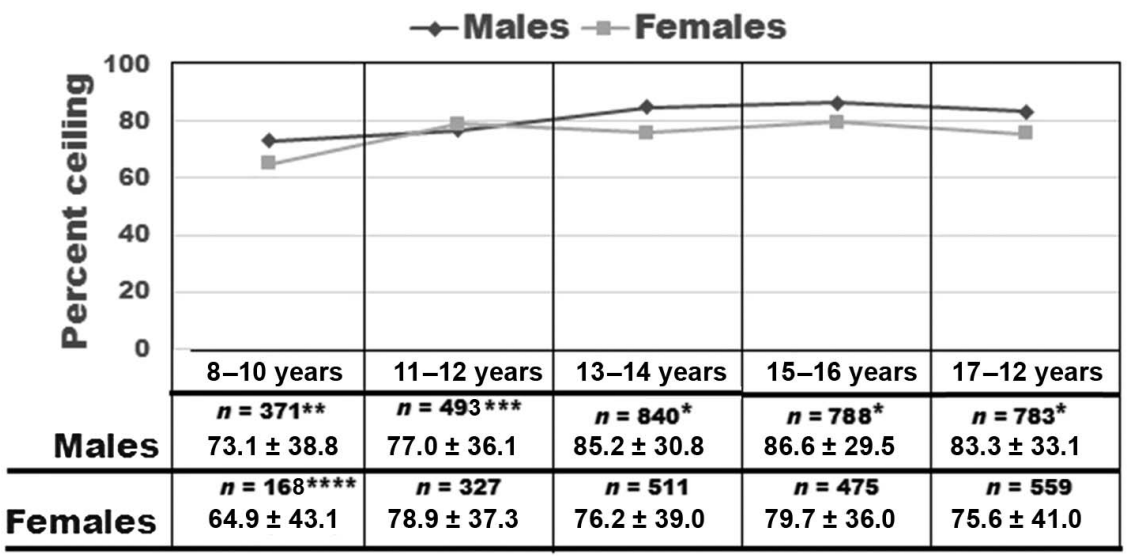

Figure 3 - BLC subtest for combined items (6) for each age group. *Males significantly $(p<.001)$ higher than females in the age groups: 13-14, 15-16, and 17-21 years, respectively. $* *$ Males in the 8 - to 10 -year age group significantly $(p<.001)$ lower than males in the age groups: 13-14, 15-16, and 17-21 years, respectively. $* * *$ Males in the 11- to 12 -year age group significantly $(p<.001)$ lower than males in the 15 - to 16 -year age group. $* * * *$ Females in the 8 - to 10 -year age group significantly $(p<.001)$ lower than females in the 15- to 16 -year age group. BLC = bilateral coordination. 
Males: The 8- to 10-year age group was significantly lower than 13-14, 15-16, and 17-21 years, and the 11- to 12-year age group was significantly lower than the 15- to 16-year age group.

Females: The 8- to 10-year age group was significantly lower than the 15- to 16-year age group.

Sexes: Male in the age group of 13-14, 15-16, and 17-21 years were significantly higher than their respective female age groups.

In summary, the acquisition of motor proficiency over time was seen, especially for females, in that the 8- to 10-year group consistently scored lower than the other age groups.

\section{Sex $\times$ Combined Age Groups $\times$ Subtest Items}

- ULC: Results for ULC are found in Figure 4.

Males: ULC-4 (catching the tossed ball—one hand) \%Ceiling mean was significantly lower than all other subtest item \%Ceiling means. ULC-6 (dribbling a ball-alternate hands) \%Ceiling mean was significantly lower than ULC-1 (dropping and catching ball with both hands), ULC-2 (catching tossed ball—two hands), ULC-3 (dropping and catching ball—one hand), and ULC-5 (dribbling a ball—one hand) \%Ceiling means. ULC-5 \%Ceiling mean was lower than ULC-1 and ULC-3 \%Ceiling means. No significant differences were seen among ULC-1, ULC-2, and ULC-3 \%Ceiling means.

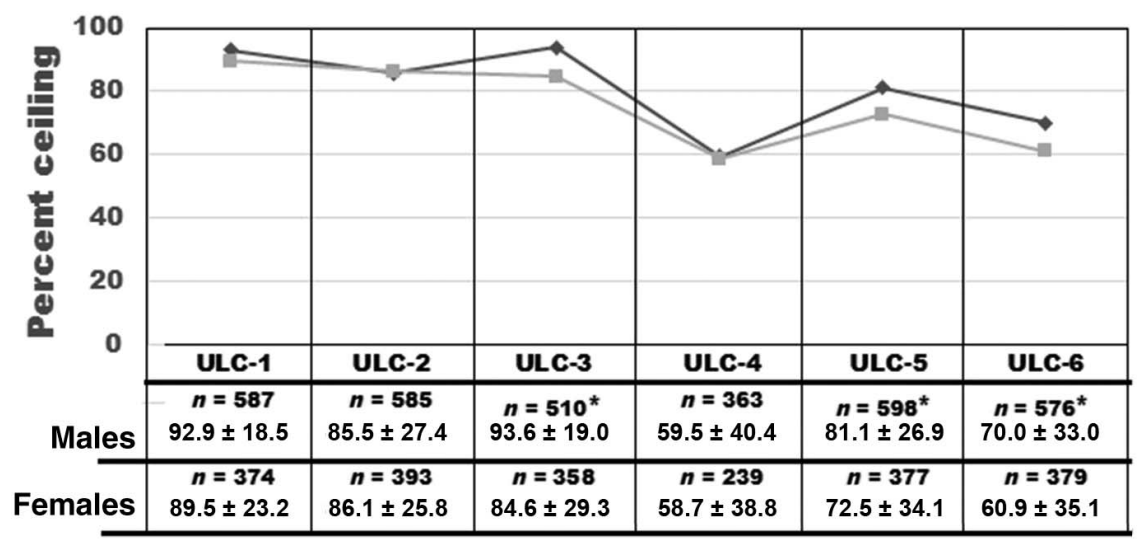

Figure 4 - ULC subtest items: percent ceiling means for combined ages (8-21 years). *Males significantly $(p<.001)$ higher than females for ULC-3, ULC-5, and ULC-6. Male subtest items: ULC-4 significantly $(p<.001)$ lower than ULC-1, ULC-2, ULC-3, ULC-4, and ULC-6; ULC-6 significantly $(p<.001)$ lower than ULC-1, ULC-2, ULC-3, and ULC-5; ULC-5 significantly $(p<.001)$ higher than ULC-4 and ULC-6. Female subtest items: ULC-4 and ULC-6 significantly $(p<.001)$ lower than ULC-1, ULC-2, ULC-3, and ULC-5; ULC-5 significantly $(p<.001)$ lower than ULC-1, ULC-2, and ULC-3. ULC $=$ upper limb coordination. 
Females: ULC-4 and ULC-6 ceiling means were significantly lower than ULC-1, ULC-2, ULC-3, and ULC-5 \%Ceiling means. ULC-5 \%Ceiling mean was significantly lower than ULC-1, ULC-2, ULC-3 \%Ceiling means. No significant differences were seen among ULC-1, ULC-2, and ULC-3, and no significant difference was seen between ULC-4 and ULC-6.

Sexes: Males demonstrated significantly higher scores than females for onehand catching (ULC-3) and ball dribbling skills (ULC-5 and ULC-6).

In summary, ULC subtest consists of activities designed to measure visual tracking with coordinated arm and hand movement (Bruininks \& Bruininks, 2005). For both sexes, results indicate that the highest performance scores for ULC items were seen when dropping and catching a ball with one or two hands and when catching a tossed ball with two hands. Lower performance scores were seen in dribbling a ball, especially with alternate hands. The lowest scores were observed when catching a tossed ball with one hand. Males scored significantly higher in three of the six ULC items.

- BAL: Results for BAL are found in Figure 5.

Males: BAL-1 (standing feet apart on a line-eyes open) and BAL-2 (walking forward on a line) \%Ceiling mean scores were significantly higher than BAL-3 (standing on one leg-eyes open), BAL-4 (standing feet apart on a line-eyes closed), BAL-5 (walking forward heel-to-toe on a line), BAL-6 (standing on one leg-eyes closed), and BAL-7 (standing on one leg on a BAL beam-eyes open) \%Ceiling means. Significantly higher \%Ceiling mean scores were seen for BAL-3, BAL-4, and BAL-5 when compared with BAL-6 and BAL-7.

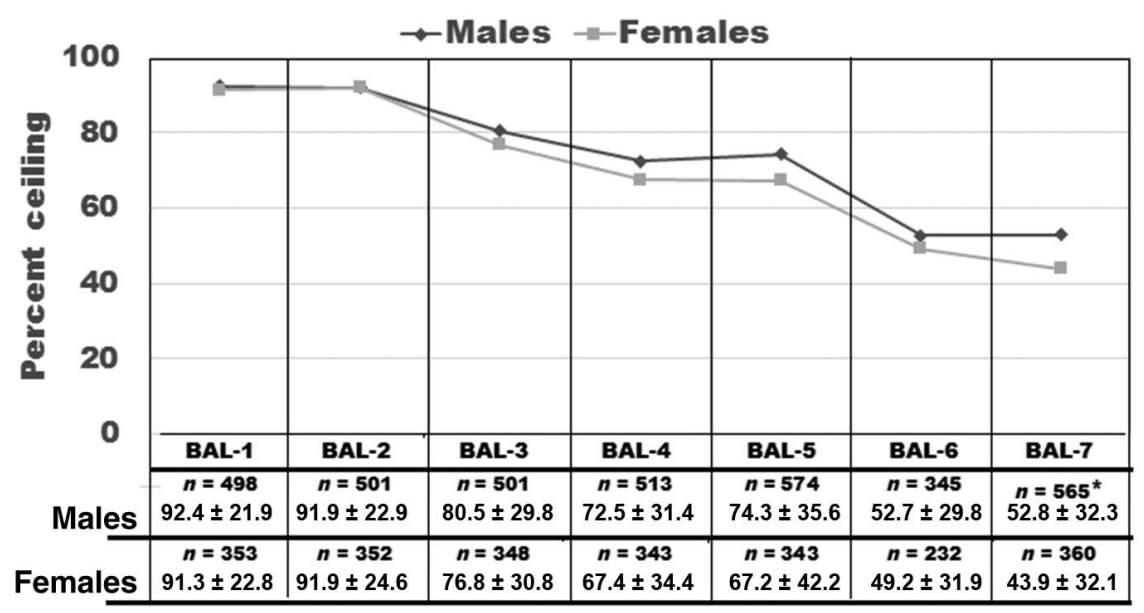

Figure 5 - BAL subtest items: percent ceiling means for combined ages (8-21 years). *Males significantly $(p<.001)$ higher than females for BAL-7. Male subtest items: BAL-1 and BAL-2 significantly $(p<.001)$ higher than BAL-3, BAL-4, BAL-5, BAL-6, and BAL-7; BAL-3, BAL-4, and BAL-5 significantly $(p<.001)$ higher than BAL-6 and BAL-7. Female subtest items: Results are the same as males. $\mathrm{BAL}=$ balance. 
Significantly higher scores were seen for BAL-1 (standing feet apart on a line-eyes open) and BAL-2 (walking forward on a line) when compared with BAL-3 (standing on one leg-eyes open), BAL-4 (standing feet apart on a line-eyes closed), BAL-5 (walking forward heel-to-toe on a line), BAL-6 (standing on one leg-eyes closed), and BAL-7 (standing on one leg on a BAL beam-eyes open). BAL-4 and BAL-5 scores were significantly higher when compared with scores for BAL-6 and BAL-7.

Females: Results were similar to those seen in males.

Sexes: Males scored significantly higher in standing on one leg-eyes closed (BAL-7).

In summary, BAL subtest evaluates motor control skills that are essential for maintaining posture when standing and walking (Bruininks \& Bruininks, 2005). Consistent findings were found for both sexes for BAL items in that (a) scores at or above $70 \%$ ceiling were observed for BAL-1 through BAL-5 and (b) lowest scores were seen for standing on one leg-eyes closed (BAL-6) and balancing on a beam on one foot (BAL-7). Males scored significantly higher than females in only one of the seven items.

- BLC: Results for BLC are found in Figure 6.

Males: BLC-4 (jumping in place-opposite sides synchronized) \%Ceiling mean score was significantly lower than all other BLC subtest items. BLC-1 (touching nose with fingers-eyes closed) and BLC-6 (tapping with same side synchronized) demonstrated significantly higher \%Ceiling mean scores than BLC-2 (jumping jacks), BLC-3 (jumping in place-same sides synchronized), BLC-4, and BLC-7 (tapping feet and fingers-opposite sides synchronized).

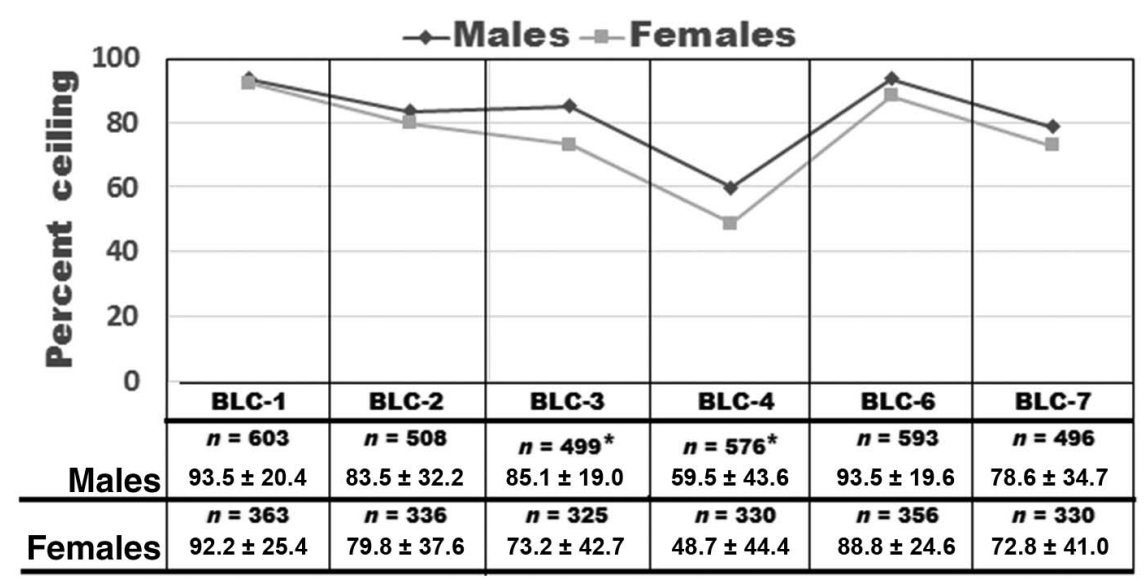

Figure 6 - BLC subtest items: percent ceiling means for combined ages (8-21 years). *Males significantly $(p<.001)$ higher than females for BLC-3 and BLC-4. Male subtest items: BLC-4 significantly $(p<.001)$ lower than all other test items; BLC-1 and BLC-6 significantly $(p<.001)$ higher than BLC-2, BLC-3, BLC-4, and BLC-7. Female subtest items: Results are the same as males. $\mathrm{BLC}=$ bilateral coordination. 
Females: Results were similar to those seen in the males.

Sexes: Males scored significantly higher for BLC-3 and BLC-4.

In summary, BLC subtest evaluates motor abilities concerned with facilitating the participation in sport and recreational activities (Bruininks \& Bruininks, 2005). Consistent findings were reported for subtest BLC between sexes. That is, higher scores $(\sim 90 \%$ ceiling) were seen for touching nose, eyes closed, and synchronized tapping of feet and fingers. A secondary set of high scores (70-80\%) was observed for jumping jacks and bilateral movement for arms and legs when movement was ipsilaterally synchronized. The lowest scores (50-60\%) were noted when performing contralateral synchronized leg-foot movements (BLC-4). Therefore, the most difficult BLC movements involved contralateral synchronization. Males scored higher than females in two of the six items.

\section{Sex $\times$ Combined Age Groups $\times$ Combined Subtests}

Results of an ANOVA with post hoc Scheffe from summary data of combined age groups (8-21 years) and combined subtest (six for BLC, seven for BAL, and six for ULC) for males and females is found in Figure 7. For males and females, \%Ceiling means for ULC- and BLC-combined subtest items were significantly higher than BAL. Therefore, when considering skill levels of motor proficiency/competence, both sexes score lowest in BAL skills. Males scored were significantly higher when compared with females for all subtests.

\section{Discussion}

The purpose of the BOT-2 is to screen for motor impairment and determine the need for therapeutic intervention (Bruininks \& Bruininks, 2005). It was

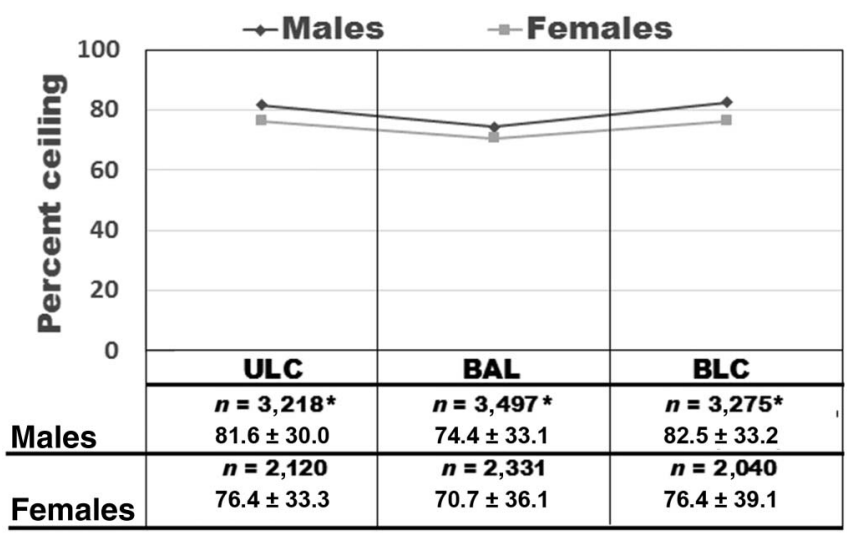

Figure 7 - Comparison of combined subtest items for combined ages (8-21 years). * Males significantly $(p<.001)$ higher than females for all subtests. Male and female subtests: BAL significantly $(p<.001)$ lower than ULC and BLC. ULC $=$ upper limb coordination; $\mathrm{BAL}=$ balance; $\mathrm{BLC}=$ bilateral coordination . 
standardized using a normative sample of 1,520 youth between the ages of 4 and 21 years living in the United States. Ceiling scores were established for each item within subtests where test administration was stopped (i.e., no impairment exists), and the evaluator moved on to the next item. In the present study, all raw scores (i. e., steps, catches, touches, jumps, dribbles, BAL time) of the participants were normalized by converting to percent of test score ceiling (\%Ceiling). Conversion of raw scores to \%Ceiling allowed for comparison between sex, age group, items within subtest, and subtest.

In addition, this method of scoring allowed for expanding the findings by Bruininks and Bruininks (2005). That is, Bruininks and Bruininks (2005) reported that youth with ID scored nearly 2 SDs lower for ULC, BAL, and BLC subtest scaled scores when compared with TD youth. However, as seen in Figures 4-6, mean item scores for these subtests ranged from $94 \%$ ceiling to $44 \%$ ceiling. Specifically, when considering the 19 items tested: (a) means greater than $80 \%$ ceiling scores were seen in eight items (males) and seven items (females), (b) means between $70 \%$ and $80 \%$ ceiling scores were reported for four items (males) and five items (females), and (c) means less than $60 \%$ ceiling scores were only reported for four items for both males and females. Accordingly, generalization of below expected motor proficiency (i.e., nearly $2 S D$ s) across subtest items cannot be assumed for this population.

In the normative tables for the BOT-2 (Bruininks \& Bruininks, 2005), similar total point scores (i.e., adding point score for each item with point score determined by raw score) for each subtest for males and females are necessary to qualify for the same specific scale score, with the scale score representing the overall fine and gross motor proficiency of a child or adolescent. A scale score for each age group is determined by the combination of all the total point scores for the eight subtests. Accordingly, for a 15 year old to qualify for a scale score of 10, females need subset scores of 22, 32, and 35 for ULC, BAL, and BLC, respectively, and males need subset scores of 22, 31, and 36 for ULC, BAL, and BLC, respectively. Therefore, little sex difference exists in scoring methodology as established by the BOT-2 norm tables for ULC, BAL, and BLC subtest (Bruininks \& Bruininks, 2005).

In the present study, when considering sex differences for age groups and subtest items, in only four items out of the 95 comparisons did males score significantly higher than females (Tables 4-6). Of interest, all four items involved catching (ULC-2 and ULC-3) or dribbling (ULC-5 and ULC-6) a ball at the youngest (8-10 years) age group. These results suggest that acquisition of ball skills equalized between sexes and remain so from late childhood into adolescents and young adulthood.

When considering sex differences for age groups when subtest scores were combined, significant sex differences were seen in one, two, and three age groups for ULC (Figure 1), BAL (Figure 2), and BLC (Figure 3), respectively. In addition, when considering sex differences for subtest items when age groups were combined, significant sex differences were seen in three, one, and two subtest items in ULC (Figure 4), BAL (Figure 5), and BLC (Figure 6), respectively. Finally, when considering sex differences when age groups and subtest items were combined, males scored significantly higher in all three subtests (Figure 7). The results of this study indicate that sufficient sex differences exist in motor proficiency across ages 
and subtest items for the BOT-2, and therefore, sex data should not be combined to established age/item-related standards for this population.

The higher ratio of males to females (2.0) reported in this study is not unique. Higher prevalence of moderate ID among male children has been reported, with males 1.6-1.9 times more likely to be classified with moderate ID than females (Croen, Grether, \& Selvin, 2001; Drews, Yeargin-Allsopp, Decoufle, \& Murphy, 1995; Richardson, Katz, \& Koller, 1986), and these sex differences seem to increase with age up to 15 years (Wen, 1997). Factors that could play a role in sex differences for ID are X-linked conditions (Chelly \& Mandel, 2001; Partington, Mowat, Einfeld, Tonge, \& Turner, 2000; Tariverdian \& Vogel, 2000), low birth weight and an association between low birth weight and low IQ in males (Matte, Bresnahan, Begg, \& Susser, 2001; Zubrick et al., 2000), and maternal smoking, which has a proportionaly higher detrimental effect on males than females (Zaren, Lindmark, \& Bakketeig, 2000).

Data provided by the BOT-2 reported a clear developmental acquisition of motor proficiency, demonstrating a progressive increase in median subtest point scores from ages 4 to 21 (Bruininks \& Bruininks, 2005, pp. 58-59). However, median subtest point scores for the BOT-2 subtest items for ULC, BAL, and BLC began leveling off at age 10 ( $>88 \%$ of median point scores), followed by small increases to age 21 . Similar results were reported in the present study. For the males, although no significant differences were seen in age groups for ULC and BAL, significantly lower scores were seen in the lower age groups (8-10 and 11-12 years) for BLC. For the females, significantly lower scores were seen across all subtests for the 8-to 10year age group. In summary, although the \%Ceiling scores were lower when compared with TD youth, the results of this study suggest a similar developmental acquisition of motor proficiency for youth with ID when compared with TD youth.

Limitations of this study reflect the characteristics of youth with ID and geographical constraints. The large variability in scores reflected by $S D$ s ranging from $20 \%$ to $40 \%$ ceiling is also consistent with previous reports on the BOT-2 (Pitetti et al., 2017) as well as other physical fitness measurements of youth with ID (Pitetti et al., 2013). Data reported in this study consist of sample participants from a limited geographical location. Whether or not these BAL and coordination capacities are reflective of the general population of children and adolescents with ID within the United States awaits research from other sectors of the country.

Results of this study indicate that for youth with ID: (a) sufficient sex differences exist in motor proficiency that sex should not be ignored when establishing BOT-2 age or item standards for ULC, BAL, and BLC subtest; (b) a substantial number of high scores (i.e., means $>80 \%$ ceiling) were reported that below expected motor proficiency when compared with TD youth cannot be assumed; and (c) the large SDs ( 20-40\%) reported indicate a substantial variability in motor proficiency.

\section{Acknowledgments}

The authors wish to thank the teachers and the staff of the Youth Education Social Summer (YESS) program, the Arc of Sedgwick County, and physical educators and the children and adolescents of the schools who participated in this study. The authors have no conflict of interest to declare. This study was not supported by any external funding. 


\section{References}

American Association on Intellectual and Developmental Disabilities. (2010). Intellectual disability: Definition, classification, and systems of supports (11th ed.). Washington, DC: Author.

Bruininks, R.H., \& Bruininks, B.D. (2005). Bruininks-Oseretsky Test of Motor Proficiency (2nd ed.). Minneapolis, MN: Pearson.

Cairney, J., Hay, J.A., Faught, B.E., \& Hawes, R. (2005). Developmental coordination disorder and overweight and obesity in children aged 9-14 y. International Journal of Obesity, 29, 369-372. PubMed doi:10.1038/sj.ijo.0802893

Cairney, J., Hay, J.A., Faught, B.E., Wade, T.J., Coma, L., \& Flouris, A. (2005). Developmental disorder, generalize self-efficacy toward physical activity, and participation in organized and free play activities. The Journal of Pediatrics, 147(4), 515-520. PubMed doi:10.1016/j.jpeds.2005.05.013

Chelly, J., \& Mandel, J.L. (2001). Mongenic causes of X-linked mental retardation. Nature Reviews Genetics, 2(9), 669-680. PubMed doi:10.1038/35088558

Cioni, M., Cocilovo, A., Di Pasquale, F., Araujo, M.B., Siqueira, C.R., \& Bianco, M. (1994). Strength deficit of knee extensor muscles of individuals with Down syndrome from childhood to adolescence. American Journal of Mental Retardation, 99, 166-174. PubMed

Croen, L.A., Grether, J.K., \& Selvin, S. (2001) The epidemiology of mental retardation of unknown cause. Pediatrics, 107(6), e86. PubMed doi:10.1542/peds.107.6.e86

Drews, C.D., Yeargin-Allsopp, M., Decoufle, P., \& Murphy, C.C. (1995). Variation in the influence of selected sociodemographic risk factors for mental retardation. American Journal of Public Health, 85(3), 329-334. PubMed doi:10.2105/AJPH.85.3.329

Elliott, D., \& Bunn, L. (2004). Motor disorders in children with intellectual disabilities. In D. Dewey \& D.E. Tupper (Eds.), Developmental motor disorders: A neuropsychological perspective (pp. 137-151). New York, NY: Guilford Press.

Hands, B., Larkin, D., Parker, H., Straker, L., \& Perry, M. (2009). The relationship among physical activity, motor competence and health-related fitness in 14-year-old adolescents. Scandinavian Journal of Medicine \& Science in Sports, 19, 655-663. PubMed doi:10.1111/j.1600-0838.2008.00847.x

Jobling, A. (1999). Attainment of motor proficiency in school-aged children with Down syndrome. Adapted Physical Activity Quarterly, 16(4), 344-361. doi:10.1123/apaq. 16.4.344

Kambas, A., Michalopoulou, M., Fatouros, I., Christoforidis, C., Manthou, E., Giannakidou, D., ... Zimmer, R. (2012). The relationship between motor proficiency and pedometer-determined physical activity in young children. Pediatric Exercise Science, 24, 34-44. PubMed doi:10.1123/pes.24.1.34

Krombholz, H. (2006). Physical performance in relation to age, sex, birth order, social class, and sport activities of preschool children. Perceptual and Motor Skills, 102(2), 477-484. PubMed doi:10.2466/pms.102.2.477-484

Levene, H. (1960). Robust tests for equality of variances. In I. Olkin (Ed.), Contributions to probability and statistics (pp. 278-292). Palo Alto, CA: Stanford University Press.

Livingstone, G., \& Birst, P. (1986). Orthopedic disorders in school children with Down syndrome with special reference to the incidence of joint laxity. Clinical Orthopaedics and Related Research, 207, 74-76. PubMed

Lucas, B.R., Latimer, J., Doney, R., Ferreira, M.L., Adams, R., Hawkes, G., . . E Elliott, E.J. (2013). The Bruininks-Oseretsky Test of Motor Proficiency-Short Form is reliable in children living in remote Australian Aboriginal communities. BMC Pediatrics, 13, 135-147. PubMed doi:10.1186/1471-2431-13-135 
Matte, T.D., Bresnahan, M., Begg, M.D., \& Susser, E. (2001). Influence of variation in birth weight within normal range and within sibships on IQ age 7 years: Cohort study. BMJ, 323(7308), 310-314. PubMed doi:10.1136/bmj.323.7308.310

Mercer, V.S., \& Lewis, C.L. (2001). Hip abductor and knee extensor muscle strength of children with and without Down syndrome. Pediatric Physical Therapy, 13(1), 18-26. PubMed

Moore, D.S., McCabe, G.P., \& Craig, B.A. (2009). Introduction to the practice of statistics. New York, NY: W.H. Freeman and Company.

Partington, M., Mowat, D., Einfeld, S., Tonge, B., \& Turner, G. (2000). Genes on the $\mathrm{X}$ chromosome are important in undiagnosed mental retardation. American Journal of Medical Genetics Part A, 92(1), 57-61. PubMed doi:10.1002/(SICI)1096-8628 (20000501)92:1<57::AID-AJMG10>3.0.CO;2-0

Pitetti, K.H., Baynard, T., \& Agiovlasitis, S. (2013). Children and adolescents with Down syndrome: Physical fitness and physical activity. Journal of Sport and Health Science, 2(1), 47-57. doi:10.1016/j.jshs.2012.10.004

Pitetti, K.H., Miller, R.A., \& Beets, M.W. (2015). Measuring joint hypermobility using the Beighton Scale in children with intellectual disability. Pediatric Physical Therapy, 27, 143-150. PubMed doi:10.1097/PEP.0000000000000136

Pitetti, K.H., Miller, R.A., \& Loovis, M. (2017). Revisiting balance, bilateral coordination, and upper limb coordination capacities of male children and adolescents with intellectual disability. Adapted Physical Activity Quarterly, 34, 1-18. PubMed doi:10.1123/ APAQ.2016-0010

Richardson, S.A., Katz, M., \& Koller, H. (1986). Sex differences in number of children administratively classified as mildly mentally retarded: An epidemiology review. American Journal of Mental Deficiency, 91(3), 250-256. PubMed

Rivilis, I., Hay, J., Cairney, J., Klentrou, P., Liu, J., \& Faught, B.E. (2011). Physical activity and fitness in children with developmental coordination disorder: A systemic review. Research in Developmental Disability, 32, 894-910. doi:10.1016/j.ridd.2011.01.017

Robinson, L.E., Stodden, D.F., Barnett, L.M., Lopes, V.P., Logan, S.W., Rodrigues, L.P., \& D'Hondt, E. (2015). Motor competence and its effect on positive developmental trajectories of health. Sports Medicine, 45, 1273-1284. PubMed doi:10.1007/s40279015-0351-6

Stodden, D.F., Gao, Z., Goodway, J.D., \& Langendorfer, S.J. (2014). Dynamic relationship between motor skills competence and health-related fitness in youth. Pediatric Exercise Science, 26, 231-241. PubMed doi:10.1123/pes.2013-0027

Stodden, D.F., Goodway, J.D., Langerdorfer, S.J., Robertson, M.A., Rudisill, M.E., Garcia, C., \& Garcia, L.E. (2008). A developmental perspective on the role of motor skill competence in physical activity: An emergent relationship. Quest, 60, 290-306. doi:10.1080/00336297.2008.10483582

Tariverdian, G., \& Vogel, F. (2000). Some problems in the genetics of X-linked mental retardation. Cytogenetic and Genome Research, 91, 278-284. doi:10.1159/000056857

Vogt, W.P. (2005). Dictionary of statistics \& methodology: A nontechnical guide for the social sciences (3rd ed., p. 40). Thousand Oaks, CA: Sage.

Wall, A.E.T. (2004). The developmental skill-learning gap hypothesis: Implications for children with movement difficulties. Adaptive Physical Activity Quarterly, 21, 197-218. doi:10.1123/apaq.21.3.197

Watkinson, E.J., Causgrove Dunn, J.C., Cavaliere, N., Calzonetti, K., Wilhelm, L., \& Dwyer, S. (2001). Engagement in playground activities as a criterion for diagnosing developmental coordination disorder. Adapted Physical Activity Quarterly, 18(1), 18-34. doi:10.1123/apaq.18.1.18

Wen, X. (1997). The definition and prevalence of intellectual disability in Australia. Canberra: Australian Institute of Health and Welfare. 
Wrotniak, B.H., Epstein, L.H., Dorn, J.M., Jones, K.E., \& Konditis, V.A. (2006). The relationship between motor proficiency and physical activity in children. Pediatrics, 118(6), e1758-e1765. doi:10.1542/peds.2006-0742

Wuang, Y.-P., Lin, Y.-H., \& Su, C.-Y. (2009). Rasch analysis of the Bruininks-Oseretsky Test of Motor Proficiency-Second Edition in intellectual disabilities. Research in Developmental Disability, 30(6), 1132-1144. doi:10.1016/j.ridd.2009.03.003

Wuang, Y.-P., \& Su, C.-Y. (2009) Reliability and responsiveness of the BruininksOseretsky Test of Motor Proficiency-Second Edition in children with intellectual disability. Research in Developmental Disability, 30, 847-855. doi:10.1016/j.ridd. 2008.12.002

Zaren, B., Lindmark, G., \& Bakketeig, L. (2000). Maternal smoking affects fetal growth more in male fetus. Paediatric and Perinatal Epidemiology, 14, 118-126. PubMed doi:10.1046/j.1365-3016.2000.00247.x

Zubrick, S.R., Kurinczuk, J.J., McDermott, B., McKelvey, R.S., Silburn, S.R., \& Davies, L.C. (2000). Fetal growth and subsequent mental health problems in children aged 4 to 13 years. Developmental Medicine \& Child Neurology, 42(1), 14-20. doi:10.1017/ S0012162200000049 